\title{
ANTIOXIDANT ACTIVITIES OF FRACTIONS FROM ETHYL ACETATE EXTRACTS OF GARCINIA FRUTICOSA LAUTERB. LEAVES
}

\author{
RIZA SHABRINA, BERNA ELYA*, ARIKADIA NOVIANI \\ Department of Phytochemical and Pharmacognosy, Faculty of Pharmacy, Universitas Indonesia, Depok, 16424, Indonesia. \\ Email: berna.elya@farmasi.ui.ac.id
}

Received: 15 June 2018, Revised and Accepted: 20 September 2018 and 23 October 2018

\section{ABSTRACT}

Objective: This study aimed to fractionate the antioxidant activity of the ethyl acetate leaf extract and to characterize the most active fractions according to compound groups.

Methods: The ethyl acetate extract was fractionated with column chromatography using a gradient elution system. Fractions were first screened qualitatively for antioxidant activity before active fractions were quantified with respect to in vitro antioxidant activity using the 2,2-diphenyl-1picrylhydrazyl (DPPH) radical scavenging assay and the ferric-reducing antioxidant power (FRAP) assay. The compound groups were identified following separation by thin-layer chromatography.

Results: Fraction 11 exhibited the greatest DPPH radical-scavenging activity, with an IC50 value of $6.58 \mu \mathrm{g} / \mathrm{mL}$, while the fraction with the greatest antioxidant activity according to the FRAP assay was fraction 10 , with a ferric ion equivalent antioxidant activity value of $1015.34 \mu \mathrm{mol} / \mathrm{g}$.

Conclusion: Compound group identification revealed that Fractions 10 and 11 contained flavonoids, with two common to both fractions, while fraction 10 also contained one specific flavonoid.

Keywords: Antioxidant; 2,2-Diphenyl-1-picrylhydrazyl, Fractionation, Ferric-reducing antioxidant power, Garcinia fruticosa.

(C) 2018 The Authors. Published by Innovare Academic Sciences Pvt Ltd. This is an open access article under the CC BY license (http://creativecommons. org/licenses/by/4. 0/) DOI: http://dx.doi.org/10.22159/ijap.2018.v10s1.10

\section{INTRODUCTION}

Free radicals are very reactive atom which chemically reacts with other molecules [1] and may damage macromolecules, causing cell damage and homeostatic disturbance [2]. Free radicals can be neutralized by antioxidants. An antioxidant is a relatively stable molecule which can eliminate the unpaired condition of the radicals. The equilibrium between free radicals and antioxidants is important for the physiological function of the organism. If the concentration of free radicals exceeds the organism's capacity to regulate them with antioxidants and antioxidant enzymes, oxidative stress conditions will arise [2]. Many plants have been shown to identify natural antioxidants, while many groups of compounds from plants, including polyphenols and terpenoids, are known to have antioxidant properties [3].

Garcinia is a plant genus which has known potency as a source of bioactive compounds. Garcinia spp. are widely used as a food source and as traditional medicines [4]. Chemotaxonomic markers from the Garcinia genus include xantone and phloroglucinol [5]. Xanton is known to have anti-inflammatory and antioxidant activities [6], while phloroglucinol is a polyphenol compound which can also inhibit oxidative stress and inflammation [7]. There are many other compounds which have been isolated from members of the Garcinia genus, such as morelloflavone, and fukugeside from the epicarp of Garcinia brasiliensis. These compounds have also been found in other Garcinia species such as Garcinia gardeniana, Garcinia mangostana, Garcinia morella, and Garcinia dulcis. The presence of morelloflavone and fukugeside in many Garcinia species suggest that these compounds may also be chemotaxonomic markers of the Garcinia genus [8].

Many Garcinia species have been tested for antioxidant activity using various methods, such as the ferric-reducing antioxidant power (FRAP) and 2,2-diphenyl-1-picrylhydrazyl (DPPH) assays. One such example is Garcinia fruticosa Lauterb. In the only published findings on $G$. fruticosa to date, it has been reported that ethyl acetate extracts of $G$. fruticosa leaves had very high antioxidant activity toward DPPH radicals, with an IC50 score of $12.37 \mu \mathrm{g} / \mathrm{mL}$ [9]. In the current study, further tests on the antioxidant activity of $G$. fruticosa leaves were performed by fractionating ethyl acetate leaf extracts, carrying out in vitro assessments of antioxidant activity in individual fractions using DPPH and FRAP assays, and identifying the compound classes present in the most-active fractions.

\section{METHODS}

\section{Chemicals}

Ethyl acetate extract [9] from G. fruticosa leaves was supplied by the Pharmacognition and Phytochemical Laboratory of the University of Indonesia. Standards used were ascorbic acid (Sigma-Aldrich, A5960; city, state [abbreviation], USA), quercetin (Sigma-Aldrich, Q4951; city, state [abbreviation], USA), tetrandrine (Sigma-Aldrich, T2695; city, state [abbreviation], USA), and $\beta$-sitosterol (Sigma-Aldrich, B5451; city, state [abbreviation], USA).

\section{Column chromatography}

The sample used was $25 \mathrm{~g}$ ethyl acetate extract of $G$. fruticosa leaves, obtained from the previous study [9]. The extract was diluted with an equal volume of acetone. The compound was evaporated to form a dry powder. The solid phase used was $375 \mathrm{~g}$ silica gel, which was made into a suspension in $100 \mathrm{~mL} n$-hexane:ethyl acetate (100:0) and packed into the column.

Chromatography was carried out by stepwise gradient elution, with solvents of gradually increasing polarity. The mobile phase started from n-hexane:ethyl acetate 100:0, 98:2, 95:5, 90:10, 85:15, 82.5:17.5, 80:20, $75: 25,70: 30,65: 35,60: 40,55: 45,50: 50,45: 55,40: 60,35: 65,30: 70$, $25: 75,20: 80,15: 85,10: 90,5: 95$, and $0: 100$. The mobile phase was continued with ethyl acetate:methanol 95:5, 90:10, 85:15, 82.5:17.5, 
$80: 20,75: 25,70: 30,65: 35,60: 40,50: 50,40: 60,30: 70,20: 80,10: 90$, and $0: 100$. Eluate was collected in $100 \mathrm{~mL}$ fractions; each being rotary evaporated at $60^{\circ} \mathrm{C}$ to dryness, before being further resuspended.

\section{Qualitative antioxidant activity test}

Column chromatography fractions were adjusted to $1000 \mu \mathrm{g}$ dry matter/mL solvent concentration, and an aliquot was applied to a silica thin-layer chromatography (TLC) plate $60 \mathrm{~F} 254$ and eluted. The developed plate was sprayed with DPPH solution and incubated for 30 min to identify zones with antioxidant activity (visualized as yellow markings on a purple background on the plates); ascorbic acid was used as the positive control.

\section{Quantitative antioxidant activity test with the DPPH assay}

This assay was carried out using the method of Molyneux [10], with minor modifications. The antioxidant activity test was performed on fractions (dry matter adjusted to a working solution of $100 \mu \mathrm{g} / \mathrm{mL}$ in methanol) and standards $(100 \mu \mathrm{g} / \mathrm{mL}$ ascorbic acid stock solution in methanol).

An aliquot (1 mL) of a dilution series of each fraction was added to a tube blacked out with aluminum foil. This was followed by $1 \mathrm{~mL}$ DPPH solution and $2 \mathrm{~mL}$ methanol; the reaction mixture was vortexed and then incubated for $30 \mathrm{~min}$ at $37^{\circ} \mathrm{C}$, after which absorbance was measured at $516 \mathrm{~nm}$ in triplicate. The antioxidant activity from each fraction was determined by the percentage decrease in absorbance by reference to an ascorbic acid standard curve. The IC50 of each fraction was determined from the equation:

Percentage inhibition $=\frac{\text { Control absorbance }- \text { sample absorbance }}{\text { Controlabsorbance }} \times 100$

Regression of percentage inhibition versus fraction concentration allowed determination of the IC50 for that fraction. Based on the IC50 value, the antioxidant activity index (AAI) was determined according to the equation of Scherer and Godoy (2009) [11]. The AAI score was determined using the equation:

$$
\mathrm{AAI}=\frac{\text { Final concentration of DPPH }(\mu \mathrm{g} / \mathrm{mL})}{\mathrm{IC} 50(\mu \mathrm{g} / \mathrm{mL})}
$$

The antioxidant activity was categorized as low when AAI $<0.5$; moderate when $0.5 \leq \mathrm{AAI}<1.0$; strong when $1.0 \leq \mathrm{AAI}<2.0$; and very strong when $\mathrm{AAI} \geq 2$.

\section{Quantitative antioxidant activity test with the FRAP assay}

This test was based on the microplate reader method described by Bolanos de la Torre et al. (2014), using ammonium ferrous sulfate (AFS) as the standard [12]. Antioxidant activity in the FRAP method was calculated as ferrous equivalent antioxidant capacity (FeEAC) in $\mu \mathrm{mol} / \mathrm{g}$, using the equation:

$$
\text { FeEAC }(\mu \mathrm{mol} / \mathrm{g})=\frac{\Delta \mathrm{A}}{\mathrm{GRAD}} \times \frac{\mathrm{Av}}{\mathrm{Sp}_{\mathrm{V}}} \times \mathrm{D} \times \frac{1}{\text { Csample }} \times 10^{5}
$$

Where $\triangle \mathrm{A}=$ pathlength correction value, GRAD is the gradient of the AFS calibration curve, Av = aliquot volume $(300 \mu \mathrm{l}), \mathrm{Spv}=$ test sample volume $(20 \mu \mathrm{l})$, Csample $=$ sample concentration, and $\mathrm{D}=1$.

\section{Phytochemical screening}

Phytochemical screening was performed in this study to identify the compound classes present in the fractions with the highest antioxidant activities. The compound classes tested for were terpenoid, alkaloid, anthraquinone, flavonoid, tannin, and saponin. To screen for the presence of alkaloids, anthraquinones, and flavonoids, samples were loaded onto a silica TLC plate 60 F254, and separated using a specific solvent (Table 1) and detected using a particular spray (Table 2).
The saponin test was carried out by adding $15 \mathrm{mg}$ fraction to $10 \mathrm{~mL}$ hot water; the reaction mixture was then cooled and shaken hard for $5 \mathrm{~s}$, and left to stand for $5 \mathrm{~min}$. In the presence of saponins, the mixture formed a steady foam head as high as $1-10 \mathrm{~cm}$. To confirm the presence of saponins, one drop $2 \mathrm{mmol} / \mathrm{L} \mathrm{HCl}$ was added; a positive result was that the foam remained steady. In this test, the bitter melon herbal medicinal product, Momordica charantia folium, was used as a positive control. The tannin test was performed by mixing $2 \mathrm{~mL}$ fraction with 3 drops $10 \%(\mathrm{w} / \mathrm{v})$ gelatin in distilled water. The presence of tannins in the fraction was marked by the formation of a white sediment. Psidii folium extract was used as the positive control.

\section{RESULTS AND DISCUSSION}

Column chromatography of $G$. fruticosa leaf extract

A total of 466 fractions, each $100 \mathrm{~mL}$ in volume, were collected. The fractions were then assayed for antioxidant activity, using qualitative and quantitative methods, the latter involving both the DPPH and FRAP assay methods.

\section{Qualitative screening of fractions for antioxidant activity}

A total of 14 fractions (fractions 1-14) tested positive for antioxidant activity. Quantification of the antioxidant activity in each of the fractions was then determined using both the DPPH and FRAP assay methods.

Quantitative antioxidant activity assay using the DHHP method The calibration curve for the DPPH antioxidant assay is shown in Fig. 1, relating ascorbic acid concentration to percentage DPPH inhibition to ascorbic acid concentration.

Table 1: Antioxidant activity test procedure on ascorbate acid, AFS, and fraction using a microplate reader

\begin{tabular}{lllll}
\hline Ingredient & \multicolumn{3}{l}{ Volume $(\mu \mathrm{L})$} \\
\cline { 2 - 5 } & Sample & Blank & $\begin{array}{l}\text { Positive } \\
\text { control }\end{array}$ & $\begin{array}{l}\text { Blank } \\
\text { plate }\end{array}$ \\
\hline AFS/ascorbate acid/fraction & 20 & - & 20 & - \\
FRAP liquid & 280 & 280 & - & - \\
Methanol & - & 20 & - & 300 \\
Acetate buffer & - & - & 280 & - \\
Final volume & 300 & 300 & 300 & 300 \\
\hline
\end{tabular}

Shake, later incubated for $30 \mathrm{~min}$ at $37^{\circ} \mathrm{C}$. Absorption was measured at $593 \mathrm{~nm}$ wavelength

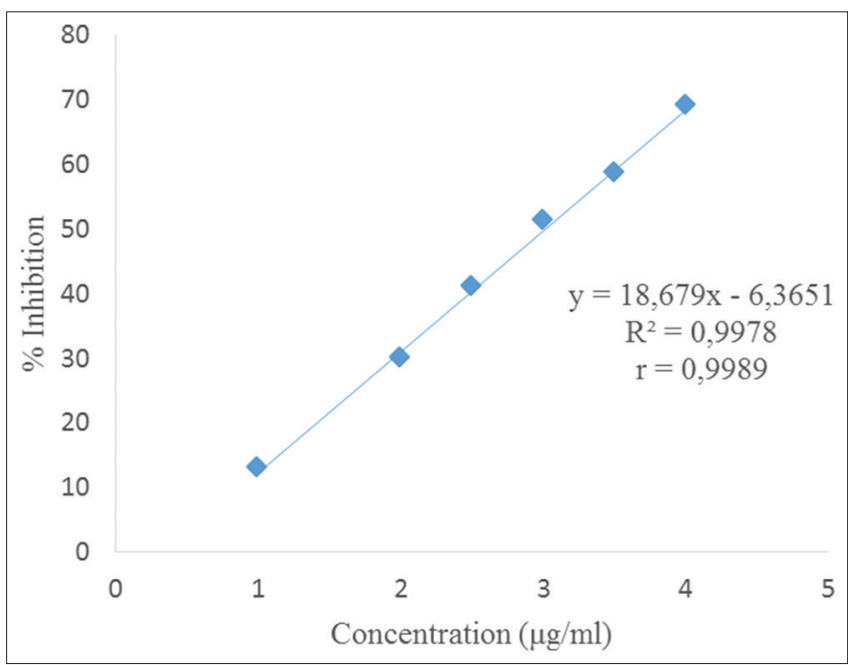

Fig. 1: The calibration curve of ascorbic acid concentration and percentage inhibition of 2,2-diphenyl-1-picrylhydrazyl antioxidant activity 
Table 2: Phytochemical screening by TLC

\begin{tabular}{|c|c|c|c|c|}
\hline $\begin{array}{l}\text { Compound } \\
\text { class }\end{array}$ & $\begin{array}{l}\text { Positive } \\
\text { control }\end{array}$ & $\begin{array}{l}\text { Spray } \\
\text { reactor }\end{array}$ & Solvent & Positive result \\
\hline Alkaloid & Tetrandrine & Dragendorff & Chloroform - methanol (8.5:1.5) & Light orange spots \\
\hline Anthraquinone & Rhei radix & Borntrager & Hexane - ethyl acetate $(1: 5)$ & Red spots \\
\hline Flavonoid & Quercetin & AlCl3 0.5\%. & $\begin{array}{l}\text { Chloroform: acetone: formic } \\
\text { acid }(7: 3: 1)\end{array}$ & $\begin{array}{l}\text { Under UV light of } 366 \mathrm{~nm} \text { wavelength, spots fluoresce } \\
\text { yellow }\end{array}$ \\
\hline
\end{tabular}

Table 3: Identification result on the compound class of Fraction 10 and 11

\begin{tabular}{lll}
\hline \multirow{2}{*}{$\begin{array}{l}\text { Compound } \\
\text { class }\end{array}$} & Result & \\
\cline { 2 - 3 } & Fraction 10 & Fraction 11 \\
\hline Flavonoid & + & + \\
Alkaloid & - & - \\
Terpenoid & - & - \\
Anthraquinone & - & - \\
Tannin & - & - \\
Saponin & - & - \\
\hline
\end{tabular}

The highest antioxidant activities, based on the DPPH assay, were detected in fractions 10-13 (Fig. 2). Fraction 10, expressing the highest DPPH antioxidant activity, eluted with the hexane:ethyl acetate solvent range from 45: 55 to 30:70, indicating that the compounds eluted were relatively non-polar.

The dosage response of antioxidant activity to fraction 11 concentration is shown in Fig. 3.

\section{Antioxidant activity assay with the FRAP method}

Ascorbic acid was used as the standard. Antioxidant activity assayed by the FRAP method was $2610.04 \mu \mathrm{mol} / \mathrm{g}$. When the same fractions which were tested with the DPPH assay (Fig. 2) were assayed with the FRAP assay (Fig. 6), the results were broadly similar (fractions 10-13 were by far the most active fractions with both assays), but clear differences were apparent. Using the DPPH assay, fractions 10-13 had very similar activities whereas, using the FRAP assay, fractions 10 and 12 were the most active, followed by fractions 11 and 13, with fraction 14 exhibiting an activity similar to that of fraction 13 .

Compound class identification in the most-active fractions

Identification of compound classes was performed on the fractions exhibiting the highest antioxidant activities based on the DPPH and FRAP assays, namely Fractions 10 and 11 . The compound classes tested for were flavonoids, alkaloids, terpenoids, anthraquinones, tannins, and saponins. Result showed that fractions 10 and 11 were flavonoid positive, in that yellow fluorescence spots were detected when the developed plates were visualized with UV at $366 \mathrm{~nm}$ wavelength, as seen with the standard (Fig. 7).

The alkaloid test result showed that alkaloids were absent from Fractions 10 and 11, although the standard tetrandine was detected as orange spots at Rf 0.43 after spraying the plate with 3\% $\mathrm{AlCl} 3$ (Fig. 8). The anthraquinone identification test showed that Fractions 10 and 11 did not contain anthraquinone, as there were no red spots on the TLC lanes representing either fraction. The positive control, Rhei radix, showed red spots at Rf 0.91 (Fig. 9). Tannin identification tests showed that neither fraction 10 nor 11 contained tannins (Fig. 11), while the saponin test indicated that saponins were absent from Fractions 10 and 11 (Table 3).

\section{DISCUSSION}

Separation using column chromatography

Elution began with a non-polar mobile phase first, finishing with the most polar combination. This was done because the sample used was

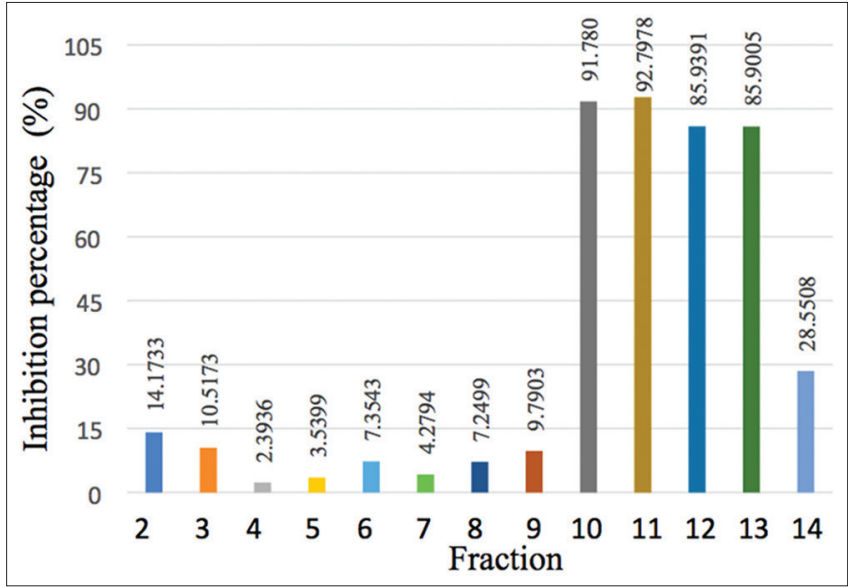

Fig. 2: Antioxidant activities, assayed by the 2,2-diphenyl-1picrylhydrazyl method, of fractions of a Gersemia fructicosa extract following column chromatography

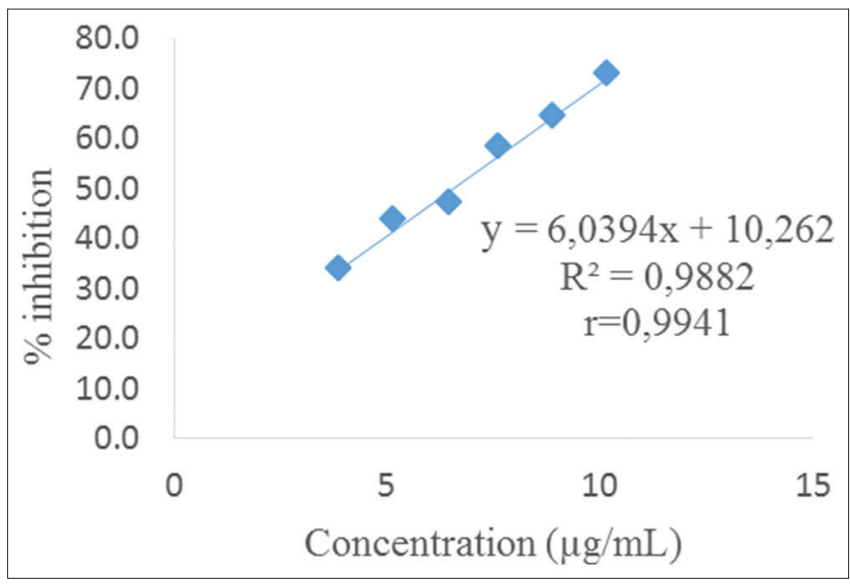

Fig. 3: The dosage-response curve of fraction 11 and percentage inhibition of 2,2-diphenyl-1-picrylhydrazyl antioxidant activity

a plant extract, which tends to contain compounds with a wide range of polarities. The use of a mobile phase with different polarities will separate the various compounds based on their polarity. From the column chromatography, there were 466 fractions, each containing $100 \mathrm{~mL}$ eluate.

\section{Qualitative screening of fractions for antioxidant activity}

Each fraction eluted from the column was tested qualitatively for antioxidant activity. Testing began with the qualitative test which aimed to identify which fractions contained antioxidant activity. Based on the results of the qualitative test, 13 fractions reduced DPPH inhibition, while one fraction (fraction 1) did not give any effect. Any fraction that exhibited antioxidant activity showed color changes when applied to a TLC plate and developed. Fractions with antioxidant activity gave white- or yellow-colored spots on a purple background when viewed under UV light with a wavelength of $366 \mathrm{~nm}$. Those fractions which 


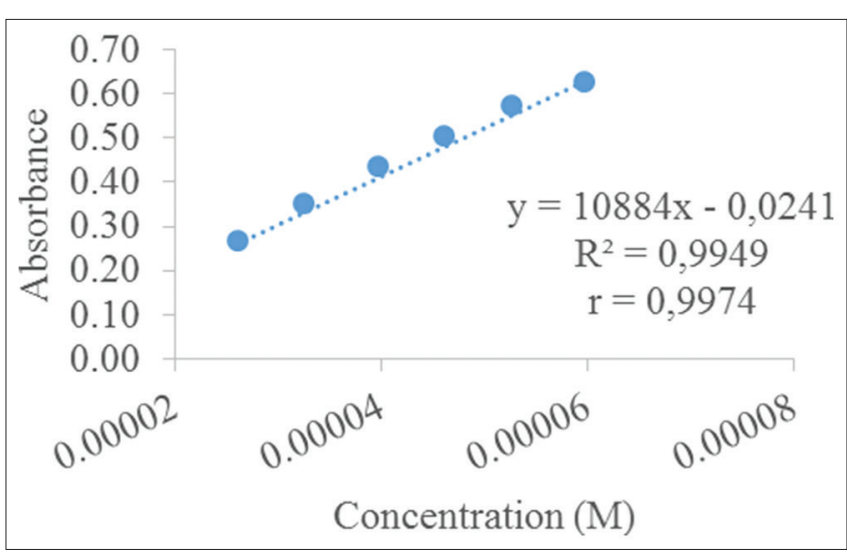

Fig. 4: Calibration curve for antioxidant activity using the ferricreducing antioxidant power assay, with ascorbic acid as the standard and absorbance measured using a spectrophotometer

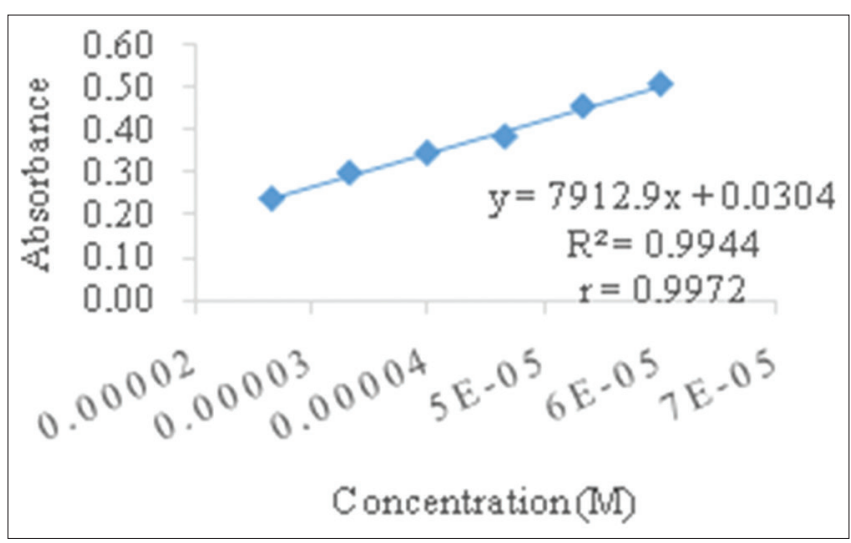

Fig. 5: Calibration curve for antioxidant activity using the ferricreducing antioxidant power assay, with ascorbic acid as the standard and absorbance measured using a microplate reader

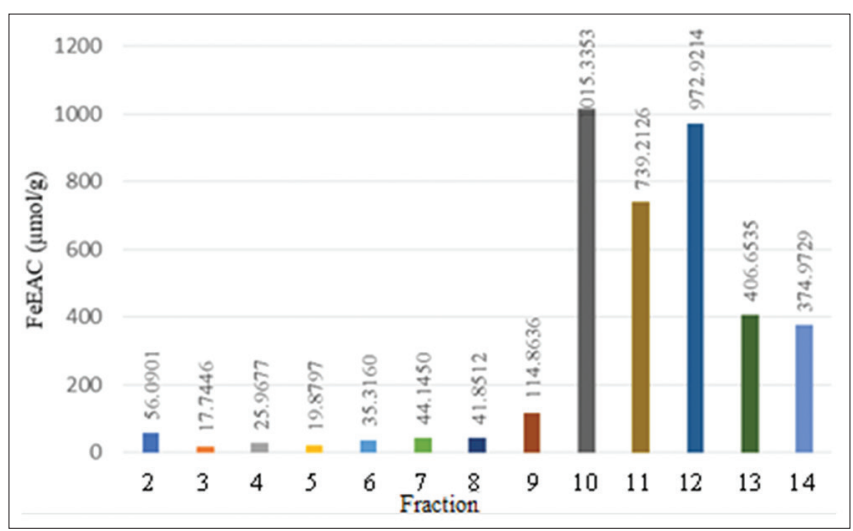

Fig. 6: Comparison of antioxidant activity in chromatographic fractions, using the ferric-reducing antioxidant power method

showed positive results with respect to the antioxidant activity in the qualitative test were then subjected to quantitative testing using the DPPH assay.

\section{Quantitative antioxidant activity test with the DPPH method}

The DPPH free radical capture method is easy to use, has high sensitivity, can analyze samples in a short time, and is widely used to measure the ability of a compound to reduce concentrations of free radicals or hydrogen donors. The solvent used is methanol. During the test, the tubes containing DPPH were wrapped in aluminum foil, because light exposure was one of the factors that decreased the stability of DPPH solutions [13]

The quantitative antioxidant activity test using the DPPH radical damping method was preceded by the determination of the maximum wavelength ofDPPH. The optimum results of DPPH wavelength indicated that the maximum absorption of the DPPH solution lay at a wavelength of $516 \mathrm{~nm}$. The measurement of uptake in the DPPH radical damping method was then performed at that wavelength. When absorbance was carried out at the optimal wavelength, the measurement was done at the peak, so if there was a slight wavelength shift, then the absorbance change would be very small. After optimization of the DPPH wavelength, antioxidant activity testing on ascorbic acid was used to calibrate the results, so that subsequent fraction samples could be assigned a relative antioxidant activity value.

In an antioxidant activity test, a standard or positive control should be included, to check that the test procedure was working properly [10]. The standard used in this test was ascorbic acid, due to its excellent radical capture and reducing agent characteristics, in accordance with the reaction mechanisms of DPPH and FRAP testing. The absorbance measurements were carried out at the optimum wavelength, at $516 \mathrm{~nm}$ for each concentration of the solution. The IC50 value for ascorbic acid in this study was $3.0176 \mu \mathrm{g} / \mathrm{mL}$, whereas published IC50 values for ascorbic acid from previous studies ranged from $0.6 \mu \mathrm{g} / \mathrm{mL}$ [11] to $6.1 \mu \mathrm{g} / \mathrm{mL}$ [12]. Differences in test results might be due to differences in test conditions, such as the concentrations of DPPH and solvents used.

The quantitative antioxidant activity tests were carried out on fractions 2-14, in response to the results from the qualitative tests. Of the fractions which tested positive for antioxidant activity using the qualitative test, the fraction with the greatest antioxidant activity (using the DPPH assay) was fraction 10, which was obtained from the eluent n-hexane-ethyl acetate in the ratio of 45:55-30:70. Fraction 10 was the most-active fraction because the original sample was an ethyl acetate extract of $G$. fruticosa leaves so that the extract contained mostly semipolar compounds.

The antioxidant activity of each fraction was determined based on the decrease in absorption of the test solution on the DPPH blank. The purple color of DPPH solution will fade to yellow due to DPPH reduction $\bullet$ to DPPH2 due to $\mathrm{H}$ atoms from antioxidants. The results were expressed in percentage of inhibition. The greater the percentage of inhibition, the greater the ability of the sample to reduce DPPH radicals. The results of the test and calculation of the percentage of inhibition for each fraction can be seen in Fig. 2. Based on the calculation, the fraction which achieved the greatest percentage inhibition was Fraction 11, equivalent to $92,7978 \%$. In addition to Fraction 11, several other fractions also exhibited a large percentage of inhibition, namely Fractions 10, 12, and 13. In this study, IC50 was calculated for only 11 fractions, and this was followed by the identification of the class of compound groups contained in each antioxidant-rich fraction. The IC50 value of Fraction 11 was $6.58 \mu \mathrm{g} / \mathrm{mL}$. AAI values for each fraction were calculated from the IC50 results obtained. The AAI value of Fraction 11 was 4.09 so that the antioxidant activity of this fraction fell into the very strong category (AAI >2). The results of the antioxidant activity test of fraction 11 can be seen in Fig. 3 .

The DPPH test is often used for antioxidant activity testing because it is easy, fast, and the only equipment required is a UV-Vis spectrophotometer. On the other hand, the DPPH test also has deficiencies, including test times. The rapid completion of the test ignores the radical dampening activity of slow-working antioxidants, where the reaction would only be completed many hours later [16]. Other deficiencies of this assay are related to steric accessibility. Fast-acting antioxidants dealing with peroxyl radicals can react slowly or even be inert to DPPH due to steric stability. DPPH will also be reduced in color by reducing agents and also by hydrogen ion transfer so that interpretation of DPPH-related antioxidant activity can become inaccurate [17]. 


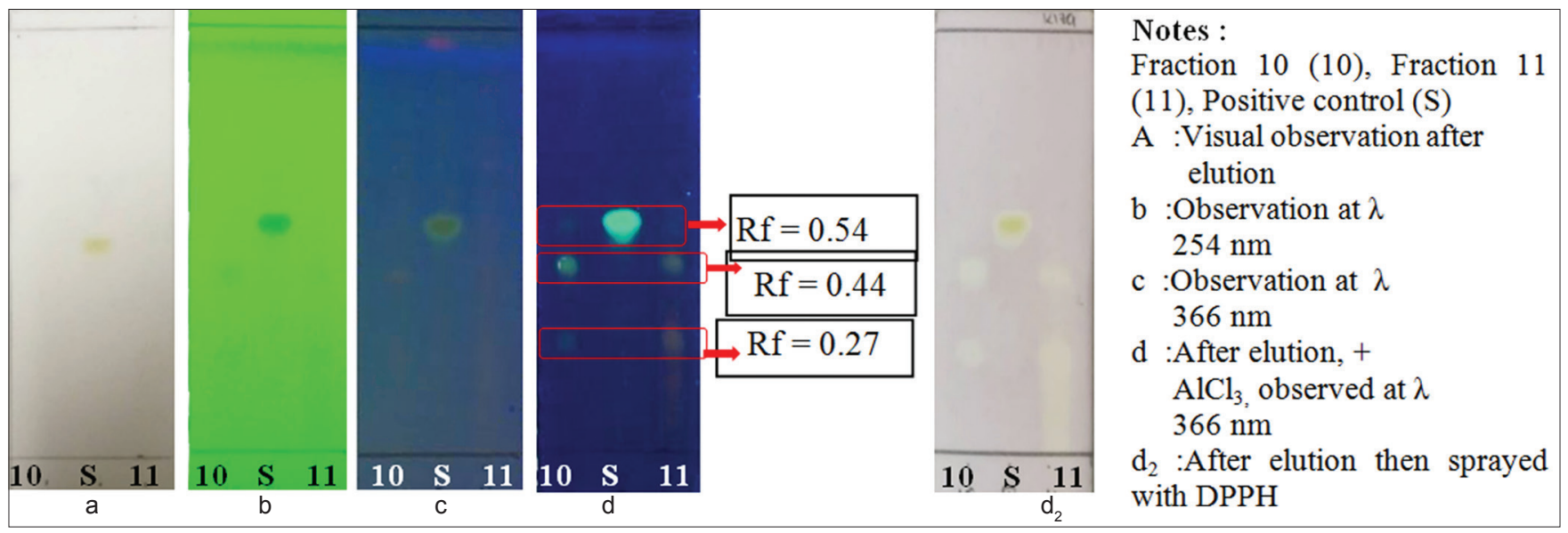

Fig. 7: Flavonoid test result

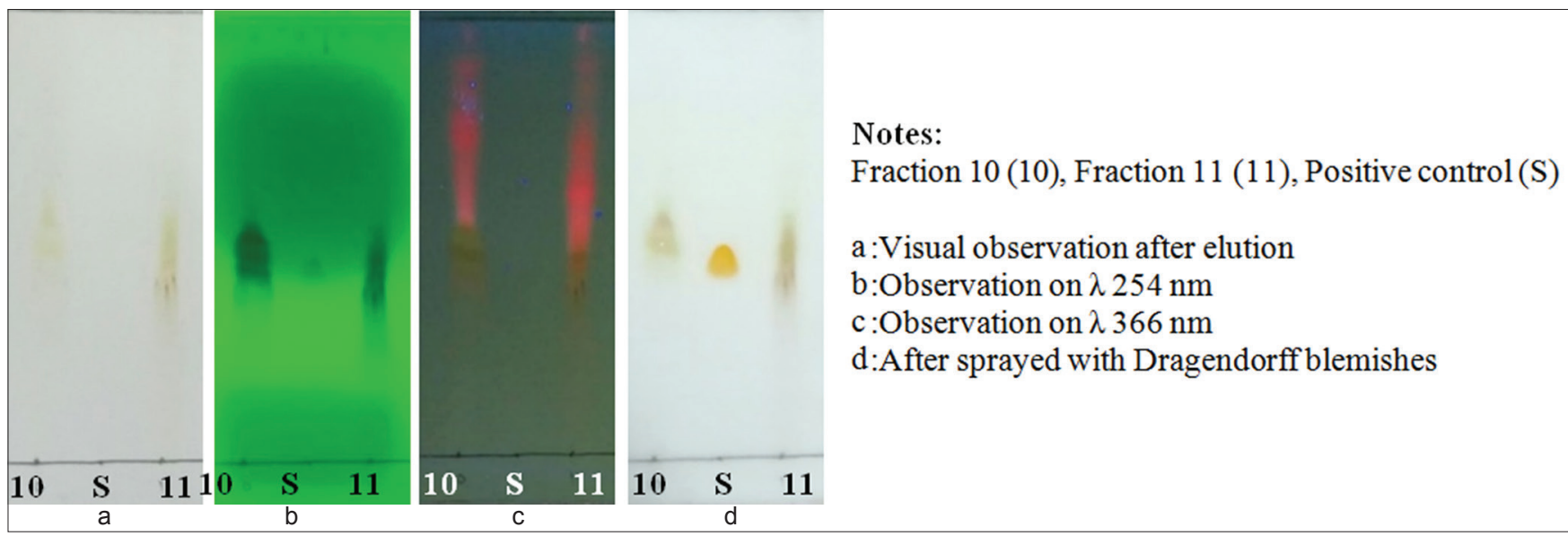

Fig. 8: Alkaloid test result
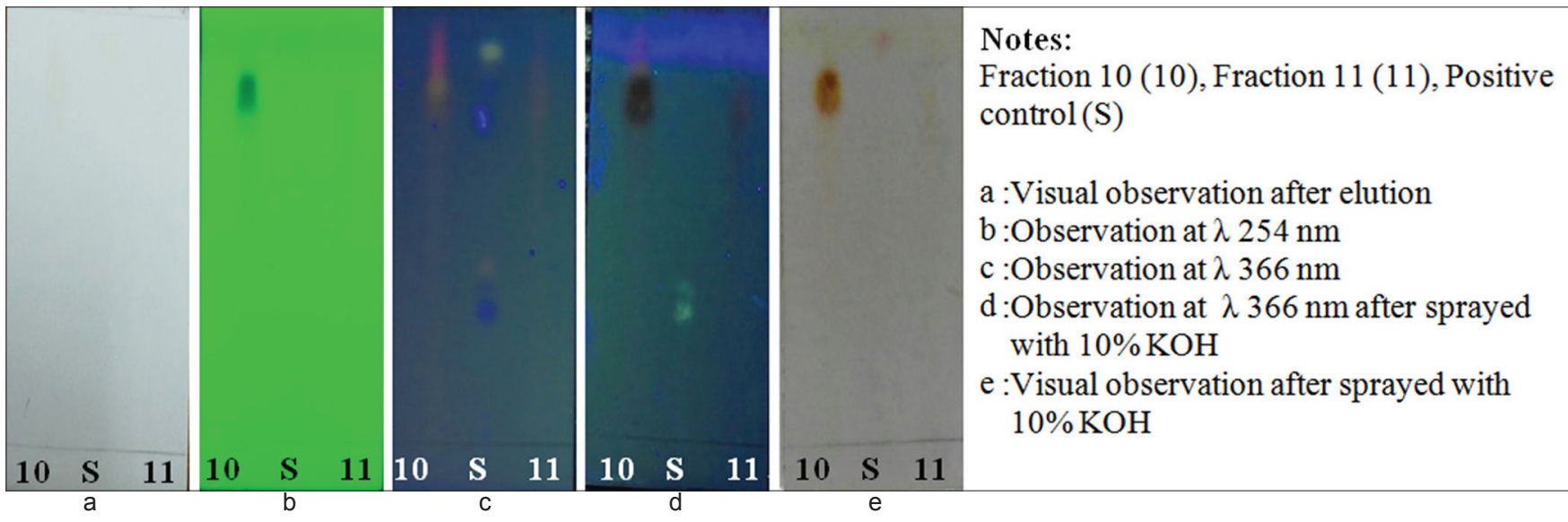

Fig. 9: Anthraquinone test result

\section{Antioxidant activity test using the FRAP method}

This test was performed using a microplate reader. A microplate reader requires a much smaller sample volume and less reagent than the more usual UV-Vis spectrophotometer. In addition, with the use of a microplate reader, all samples can be tested simultaneously so that testing time is relatively fast. The antioxidant activity assay with this microplate-reader-based FRAP method is quite easy, fast, and relatively cheap.

The path length of light on the microplate reader will be smaller in number than the $1 \mathrm{~cm}$ pathlength in a spectrophotometer. The testresults with microplate needed to be normalized so that the data obtained were comparable with the test using a UV-Vis spectrophotometer with a $1 \mathrm{~cm}$ pathlength. The microplate reader used in this study, Versamax (Supplier, city, country), did not have automatic photometric pathlength correction, so normalization was done manually.

AFS was used as a calibration standard to obtain the value of antioxidant activity in the form of iron equivalence. In addition, AFS was also used to obtain correction pathlength values. First, AFS was tested using a UVVis spectrophotometer. The test result was made into an absorbance versus concentration calibration curve so that the linear regression equation (Fig. 4) was obtained. Based on the obtained equation, the gradient value was 10884 . The same AFS solution was then tested 


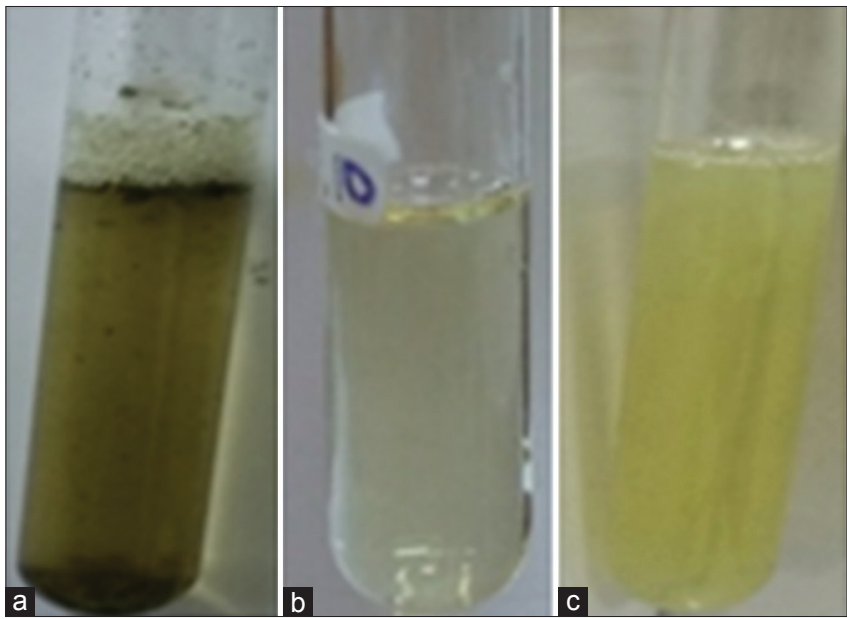

Fig. 10: Saponin test result. Momordica charantia folium (a) fraction 10 (b), and fraction 11 (c)

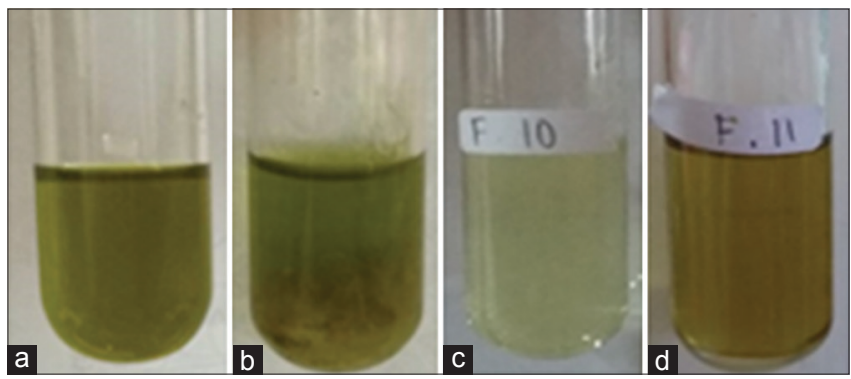

Fig. 11: Tannin test result. The standard before $10 \%$ gelatin was added (a), Psidii folium (b), fraction 10 (c), and fraction 11 (d).

using a microplate reader. Test results with the microplate reader also made concentration versus absorbance curves (Fig. 5). The gradient value was 7912.9. From the two gradients obtained, we could calculate the correction pathlength value, which was 0.73 (7912.9/10884). This value was different from the value which had been determined by Bolanos et al., which was 0.83 . In a study conducted by Wong et al., the correction pathlength value obtained was 0.76 [17]. Differences in test results could be due to differences in solvents and instruments used. Ascorbic acid was used as the standard. The test was performed using a microplate reader, and the value of antioxidant activity in FRAP method was expressed in FeEAC. The result was $2610.04 \mu \mathrm{mol} / \mathrm{g}$.

Fractions 2 to 14 were tested using a microplate reader. The fraction with the greatest antioxidant activity value, based on FeEAC calculation, was Fraction 10 with a value of $1015.34 \mu \mathrm{mol} / \mathrm{g}$. Fraction $12 \mathrm{had}$ an FeEAC value which was not much different from Fraction 10, namely $972.92 \mu \mathrm{mol} / \mathrm{g}$. Assessing the antioxidant activity with the FRAP method is quite easy, fast, and relatively cheap. The disadvantages of this method include not being able to detect compounds that work by transferring hydrogen, such as thiol antioxidants. In addition, FRAP only measures the ability to reduce a ferric ion, a characteristic which is not relevant to antioxidant activity (Fig. 6) [15].

The fraction with the highest activity on the FRAP test was different from the results in the test using DPPH. Differences in test results between the assays are due to differences in reaction mechanisms in the two tests. In the DPPH test, the ability of the compound to reduce the DPPH radical, especially with the transfer of hydrogen from the compound to the DPPH radical, was measured. In the FRAP test, electron transfer occurs, but FRAP measures only the ability to reduce, based on ferric ions. This difference in results (Figs. 2 and 6) denotes that there are fractions that contain more compounds that work with electron transfer, and there are also fractions that contain antioxidants which reduce ferric ions.

\section{Compound class identification of the compounds in the fractions} with the highest antioxidant activities

Identification was done on the fractions exhibiting the highest antioxidant activities according to radical damping tests using DPPH and FRAP, which were Fractions 11 and 10. The compound classes tested were flavonoid, alkaloid, anthraquinone, terpenoid, tannin, and saponin.

The test results showed that Fractions 10 and 11 contained flavonoids characterized by the presence of yellowish fluorescent spots at a wavelength of $366 \mathrm{~nm}$ as observed in the standard (Fig. 7). The Rf of the standard was 0,54 ; fraction 10 was 0,$27 ; 0,44$; and 0,54 ; fraction 11 was 0,27 and 0,44 . One spot infraction 10 exhibited an Rf similar to that of the quercetin spot, which had an Rf value of 0.54 . To determine which spots exhibited antioxidant activity, aliquots of fractions and standards were loaded on to TLC plates which were run then sprayed with DPPH. Spots on lanes where the samples were fractions 10 and 11 indicated that the antioxidant activity coincided with flavonoid-positive spots at $\mathrm{Rf}$ of 0.27 and 0.44 . A spot that had an Rf equal to that of quercetin did not show antioxidant activity. It showed that Fractions 10 and 11 contained flavonoid compounds, but that none of them were quercetin.

Alkaloid test results showed that fractions 10 and 11 contained no alkaloids. After spraying with $\mathrm{AlCl} 33 \%$, orange color appears on the standard tetrandrine, with a Rf of 0.43 , whereas the orange color did not appear in Fractions 10 or 11 (Fig. 8). The anthraquinone test showed that Fractions 10 and 11 did not contain anthraquinone, because there were no red spots in the fraction lanes, although the positive control, Rhei radix, showed a red spot with a Rf 0.91 (Fig. 9). The saponin test showed that Fractions 10 and 11 did not contain saponins (Fig. 10). This is because saponins are very polar compounds, while the solvent used in this study to extract the G. fruticosa leaves was ethyl acetate, which is a semi-polar solvent. In addition, the fractions tested were semi polar fractions; hence, if there was saponin in the extract, such compounds would not have been extracted or eluted from the silica column. Fractions 10 and 11 also did not contain tannins (Fig. 11). Tannins are high molecular mass compounds, which would not elute during fractionation.

\section{CONCLUSION}

The fraction of the ethyl acetate extract of $G$. fruticosa leaves which had the highest antioxidant activity based on the DPPH test was Fraction 11 with an IC50 value of $6.58 \mu \mathrm{g} / \mathrm{mL}$, whereas the fraction with the highest antioxidant activity based on the FRAP test was FRACTION 10, with an FeEAC value of $1015.34 \mu \mathrm{mol} / \mathrm{g}$. Fractions 10 and 11 of the ethyl acetate extract of $G$. fruticosa leaves contained flavonoid class compounds.

\section{CONFLICTS OF INTEREST}

All authors have none to declare.

\section{REFERENCES}

1. Lü JM, Lin PH, Yao Q, Chen C. Chemical and molecular mechanisms of antioxidants: Experimental approaches and model systems. J Cell Mol Med 2010;14:840-60.0

2. Lobo V, Patil A, Phatak A, Chandra N. Free radicals, antioxidants and functional foods: Impact on human health. Pharmacogn Rev 2010;4:118-26

3. Do QD, Angkawijaya AE, Tran-Nguyen PL, Huynh LH, Soetaredjo FE, Ismadji S, et al. Effect of extraction solvent on total phenol content, total flavonoid content, and antioxidant activity of Limnophila aromatica. J Food Drug Anal 2014;22:296-302.

4. Katrin EB, Mun'im A, Hasiholan A, Marlin I, Mailandari M. Antioxidant activities of leaves extracts of three species of Garcinia. Int J Med Aromat Plants 2012;2:691-3.

5. Ritthiwigrom T, Laphookhieo S, Pyne SG. Chemical constituents and biological activities of Garcinia cowa Roxb maejo. Int J Sc Technol 
2013;7:212-31.

6. Seruji NMU, Khong HY, Kutoi CJ. Antioxidant, anti-inflammatory, and cytotoxic activities of Garcinia nervosa (Clusiaceae). J Chem 2013;2013:1-5

7. Kim MM, Kim SK. Effect of phloroglucinol on oxidative stress and inflammation. Food Chem Toxicol 2010;48:2925-33.

8. Gontijo VS, de Souza TC, Rosa IA, Soares MG, da Silva MA, Vilegas W, et al. Isolation and evaluation of the antioxidant activity of phenolic constituents of the Garcinia brasiliensis epicarp. Food Chem 2012;132:1230-5.

9. Nurrianti J, Elya B, Mauldina MG. Inhibition of alpha-glucosidase and antioxidant test using the DPPH method of leaf extracts of Garcinia fruticosa Lauterb, and phytochemical screening on the most-active extract. Int J ChemTech Res 2017;10:207-15.

10. Molyneux P. The use of the stable free radical diphenylpicryl-hydrazyl (DPPH) for estimating antioxidant activity. Songklanakarin J Sci Technol 2004;26:211-9.

11. Scherer, R, Godoy, H. Antioxidant activity index (AAI) by the 2,2-diphenyl-1-picrylhydrazyl method. Food Chemistry 2009;112:654-8.

12. Nariya PB, Bhalodia NR, Shukla VJ, Acharya R, Nariya MB. In vitro evaluation of antioxidant activity of Cordia dichotoma (Forst f.) bark. Ayu 2013;34:124-8

13. Dontha S. A review on antioxidant methods. Asian J Pharm Clin Res 2016;9:14-32.

14. Deepak J, Anurekha J. Development of polyherbal with antioxidant activity. Asian J Pharm Clin Res 2018;11:483-5.

15. Kusmardiyani S, Novita G, Fidrianny I. Antioxidant activities from various extracts of different parts of Kelakai (Stenochlaena palustris) growth in central Kalimantan-Indonesia. Asian J Pharm Clin Res 2016;9:215-9.

16. Ozcelik B, Lee JH, Min DB. Effects of light, oxygen, and $\mathrm{pH}$ on the absorbance of 2, 2-Diphenyl-1-picrylhydrazyl. J Food Sci 2003;68:487-90

17. Apak R, Özyürek M, Güçlü K, Çapanoğlu E. Antioxidant activity/ capacity measurement. 2. hydrogen atom transfer (HAT)-based, mixedmode (electron transfer (ET)/HAT), and lipid peroxidation assays. J Agric Food Chem 2016;64:1028-45.

18. Prior RL, Wu X, Schaich K. Standardized methods for the determination of antioxidant capacity and phenolics in foods and dietary supplements. J Agric Food Chem 2005;53:4290-302. 